\title{
Experimental Study of Modified Videman Method to Replicate Knee Osteoarthritis in Rabbits
}

\author{
LIU Jing', LIN Qiaoxuan², LU Liming ${ }^{2}$, GUO Zexing ${ }^{2}$, LIU Hong ${ }^{1,3}$, ZHANG Liangzhi ${ }^{1,3}$, XIU Zhongbiao ${ }^{1,3,4^{*}}$ \\ ${ }^{I}$ The People's Hospital Affiliated of Fujian University of Traditional Chinese Medicine, Fuzhou, Fujian 350004, China; \\ ${ }^{2}$ College of Traditional Chinese Medicine, Fujian University of Traditional Chinese Medicine, Fuzhou, Fujian 350122, China; \\ ${ }^{3}$ Key Laboratory of Orthopedics \& Traumatology of Traditional Chinese Medicine and Rehabilitation, Ministry of Education, Fuzhou, \\ Fujian 350122, China; \\ ${ }^{4}$ Fujian Institute of Orthopaedics, Fuzhou, Fujian 350004, China \\ *Correspondence: XIU Zhongbiao, E-mail: xzbdoctor@sina.com
}

\begin{abstract}
Objective: To establish a convenient, stable and efficient rabbit model of knee osteoarthritis (KOA). Methods: Sixty male New Zealand rabbits were selected as the normal group, traditional Videman method group (traditional group) and modified Videman method group (improved group) according to the random number table method, with twenty cases in each group. In the traditional group, the left knee joint was straightened for 6 weeks with ordinary tubular plaster. In the improved group, a plaster support was placed on the front of the knee and wrapped a single layer of polymer plaster in a ring. The left knee joint of the rabbit was fixed in a straight position for 6 weeks for modeling, according to the modeling principle of the traditional group. During the process of modeling, the general conditions of the falling off of the plaster, blood supply to the left hind limb and death were recorded. Lequesne MG knee grade score method was used to evaluate the left knee joint motor function of rabbits after modeling. The joint space, effusion, cartilage surface smoothness were evaluated by X-ray and MRI. The macropathology of cartilage and synovium, hematoxylin-eosin staining (HE staining) were observed by light microscope and Mankin's scores were applied to investigate morphological changes. The average time taken to fix and disassemble the plaster was compared with that of the traditional and improved groups. Results: 1$)$ Comparison of the general conditions of modeling: there was no death in the normal group. In the traditional group, there were 6 cases which plaster were slipped and re-fixed, 4 cases died due to perineal infection and 1 case died from dstarving themselves, 3 cases that had ischemic necrosis of lower limbs, 12 cases were successfully established finally. In the improved group, the plaster was slipped and refixed in 2 cases, 1 case died of perineal infection and 1 case died of lower limb swelling and necrosis, and 18 cases were successfully modeled. The success rate of the improved group was higher than that of the traditional group $(P<0.05)$. 2) Comparison of behavioral outcomes: in the comparison with the normal group, Lequesne MG knee joint evaluation method in the traditional group and the improved group were risen $(P<0.05)$. There was no significant difference in Lequesne $\mathrm{MG}$ scores between the traditional group and the improved group $(P>0.05)$. 3) Comparison of imaging results: compared with the normal group, $X$-rays showed a narrowing of joint space between the modified group and the traditional group $(P<0.05)$. MRI showed increased fluid accumulation in the articular cavity, less smooth femoral condyle cartilage, and MOAKS score increased significantly $(P<0.05)$. There was no significant difference in imaging results between the traditional group and the improved group $(P>0.05)$. 4) Comparison of morphology: compared with the normal group, the cartilage and synovium of the improved group and the traditional group were observed to have dull cartilage surfaces, rough articular surfaces and some defects. The anterior patellar synovium was thickened and yellow, and the macropathology score of the cartilage was increased $(P<0.05)$. HE staining of cartilage was observed by light microscopy to show that the surface of the cartilage was uneven and the chondrocytes were not arranged in an orderly manner. Chondrocyte clusters were occasionally observed, and the Mankin's scores were increased $(P<0.05)$. There was no significant difference in morphology between the traditional group and the improved group $(P>0.05)$. 5) Comparison of the average time taken to fix and disassemble the plaster: the average time of the improved group was significantly shorter than that of the traditional group $(P<0.05)$. Conclusion: The KOA rabbit model established by modified Videman method has the advantages of simple operation, firm fixation, convenient disassembly and high success rate of modeling, which is conducive to the study of KOA mechanism.
\end{abstract}

KEY WORDS knee osteoarthritis; animal model; modified Videman method; cartilage morphology

DOI: $10.3724 /$ SP.J.1329.2020.03009

\section{更 正}

本刊 2020 年第 2 期第 113 页“(上接第 18 页)”应为“(上接第 102 页)”;第 144 负“(上 接第 39 页)”应为 “(上接第 123 页)”;第 161 页 “(上接第 64 页)”应为 “(上接第 148 页)”。 特此更正,并向广大读者致歉。 